\title{
Dynamic Geometry of Polygons
}

\author{
L. Richard Hitt and Xin-Min Zhang
}

Xin-Min Zhang received his undergraduate education in China, and received the M.S. and Ph.D. in mathematics from the University of Massachusetts at Amherst. Since 1990, he has been teaching at the University of South Alabama where he is now an associate professor. His research interests have been focused on geometry, topology, and inequality theory.

L. Richard Hitt received his B.S., M.S., and Ph.D. from Florida State University. $\mathrm{He}$ is a professor of mathematics at the University of South Alabama where he has been on the faculty since 1977. His research interests include geometric topology, especially knot theory, as well as elementary and differential geometry. His teaching interests also include mathematical modeling and the use of computing technology in mathematics.

\section{Introduction}

For a given $n$-sided plane polygon $P_{0}$, one may construct a sequence of polygons via an iterative procedure. A simple example of this sort of construction is the so-called Kasner polygons [20] - construct a second polygon $P_{1}$ whose vertices are the midpoints of the sides of $P_{0}$; construct a third polygon $P_{2}$ whose vertices are the midpoints of the sides of $P_{1}$; continuing this process one obtains a sequence of $n$-gons. An interesting question is: what can we say about the limit of this sequence? It is obvious that the size of the polygons will get smaller rapidly, but what about the limiting behavior of their shapes? For instance, if we consider a triangle, joining the mid-points on each side

Verbindet man die Seitenmittelpunkte eines vorgelegten Dreiecks, so erhält man ein zum Ausgangsdreieck ähnliches Dreieck. Fährt man in dieser Weise fort, so erhält man eine Folge immer kleiner werdender ähnlicher Dreiecke, welche zum Schwerpunkt des Ausgangsdreiecks konvergiert. In der vorliegenden Arbeit untersuchen L.R. Hitt und X.-M. Zhang analog konstruierte Folgen von beliebigen $n$-Ecken in der Ebene. Im Gegensatz zum Fall $n=3$ ist aber die Beschreibung des Grenzwerts der entsprechenden Folge von Polygonen für $n>3$ im allgemeinen nicht einfach. Auch wenn sich die Autoren deshalb auf das Studium von Polygonen, welche einen Umkreis besitzen, beschränken müssen, finden sie eine Fülle interessanter Ergebnisse und stellen dabei mannigfache und unvorhergesehene Zusammenhänge $\mathrm{zu}$ anderen mathematischen Gebieten her. $j k$ 
yields a similar triangle. Consequently all triangles in the resulting sequence are similar triangles. If we consider a quadrilateral, joining the midpoints on each of the four sides produces a parallelogram. All subsequent quadrilaterals are parallelograms. However, when we consider an arbitrary pentagon and produce the sequence of pentagons by this midpoint-construction, the limiting situation can be very complicated.

These kinds of questions have been investigated by many mathematicians including P.J. Davis, G.R. Maclane, A.M. Gleason, G.C. Shephard, J. Douglas, and I.J. Schoenberg $[3,4,5,7,9,20]$. Besides the Kasner polygons, constructing sequences of triangles and studying the geometry of their limits has repeatedly appeared in undergraduate math journals and books as well as in proposed problems in American Mathematical Monthly $[1,6,10,12,21]$.

When one tries to generalize the problem for triangles to $n$-sided polygons, there is a great deal of difficulty. Part of the reason might be the fact that every triangle is uniquely associated with two circles - an inscribed circle and a circumscribed circle - whereas a general $n$-sided polygon $(n \geq 4)$ does not always have this property.

In this paper, we will be concerned with the construction of sequences of polygons that are inscribed in a circle (they are called "cyclic" in this case.) We find this kind of construction and the underlying analysis and algebra to be interesting. It is known that the class of cyclic polygons plays an important role in isoperimetric problems for plane polygons. We hope that they can bridge the gap of difficulty between the constructions of sequences of triangles and sequences of general polygons.

\section{Nesting Triangles}

We will begin with some well-known simple examples.

Example 2.1 Take any scalene triangle $T_{0}$ and construct the inscribed circle. The points of tangency form a second triangle; denote it by $T_{1}$. Then construct the inscribed circle for $T_{1}$. The points of tangency on the three sides of $T_{1}$ form a third triangle $T_{2}$. Continuing this process one gets a sequence of triangles $\left\{T_{n}\right\}_{n=0}^{\infty}$. What does the shape of $T_{n}$ look like as $n$ gets bigger and bigger? The answer is that $T_{n}$ will be closer to an equilateral triangle! (Of course, if $T_{0}$ is equilateral, then every subsequent $T_{n}, n \geq 1$, will be equilateral.) To confirm the answer, observe from Figure 1 that

$$
\begin{aligned}
& A_{1}=\frac{\pi-A_{0}}{2}, A_{2}=\frac{\pi-A_{1}}{2}=\left(\frac{\pi}{2}-\frac{\pi}{4}\right)+\frac{A_{0}}{4}, \\
& A_{3}=\left(\frac{\pi}{2}-\frac{\pi}{4}+\frac{\pi}{8}\right)-\frac{A_{0}}{8}, A_{4}=\ldots, \\
& A_{n}=\pi \sum_{k=1}^{n}(-1)^{k+1} \frac{1}{2^{k}}+(-1)^{n} \frac{A_{0}}{2^{n}}, \quad \text { and } \quad \lim _{n \rightarrow \infty} A_{n}=\frac{\pi}{3} .
\end{aligned}
$$

Similarly, we have $\lim _{n \rightarrow \infty} B_{n}=\lim _{n \rightarrow \infty} C_{n}=\pi / 3$.

This problem has been discussed in $[1,12]$ using slightly different arguments. 


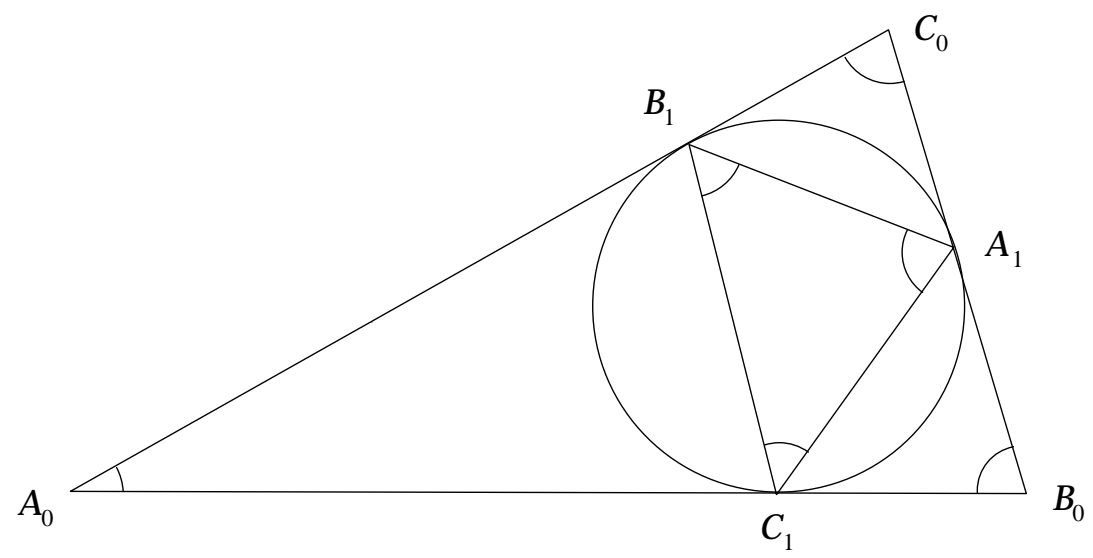

Fig. 1

Example 2.2 A variation of the first example is the following. Let $T_{0}=\triangle A_{0} B_{0} C_{0}$ be any scalene triangle circumscribing a circle $\Gamma_{0}$ with center $O$. The line segments $A_{0} O, B_{0} O$, and $C_{0} O$ (the angle bisectors of $T_{0}$ ) intersect $\Gamma_{0}$ at points $A_{1}, B_{1}$, and $C_{1}$, respectively, and form a second triangle $T_{1}=\triangle A_{1} B_{1} C_{1}$ that circumscribes a circle $\Gamma_{1}$ with center $O_{1}$. Construct a third triangle from $T_{1}$ in the same manner, and so on. We have a new sequence of triangles that are nested in a coherent manner. The triangle $T_{n}$ in this sequence is also approaching the equilateral one as $n$ increases. To see this, notice that from Figure 2

$$
A_{n}=\sum_{k=1}^{n} \frac{\pi}{4^{k}}+\frac{A_{0}}{4^{n}}, \quad n \geq 1,
$$

and the rest of the argument is the same as Example 2.1. This problem was posed in [10].

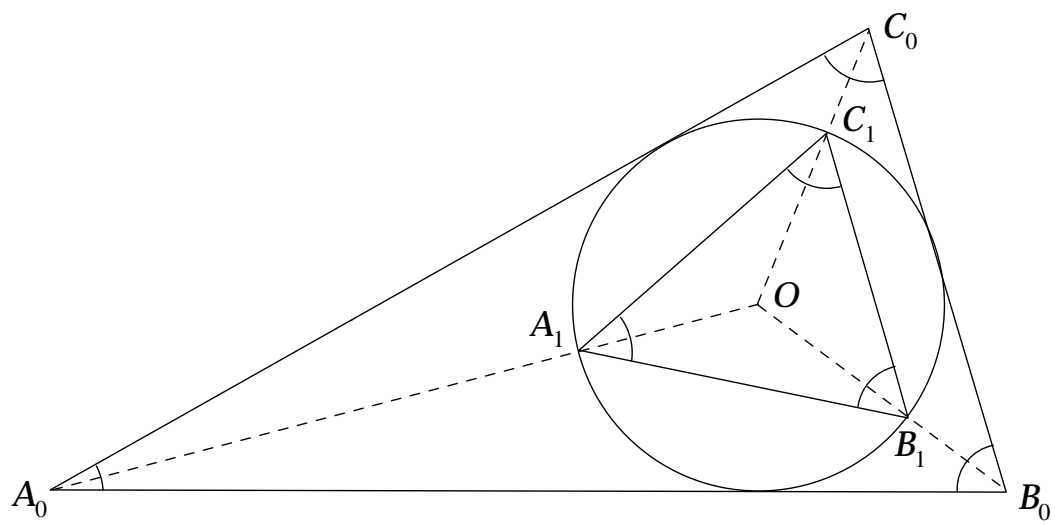

Fig. 2 


\section{Remark 2.3}

(i) There are certainly other ways to construct nesting triangles whose members become closer and closer to equilateral. For instance, let $T_{0}$ again be a scalene triangle with vertices $A, B$, and $C$, and let $T_{1}$ be the triangle formed by the intersection points of the angle bisectors of $T_{0}$ on its three sides. Construct $T_{2}, T_{3}, \ldots$ in the same way. Then it can be proved that the shape of $T_{n}$ will be closer and closer to equilateral as $n$ gets bigger and bigger. Trimble gave a proof for the special case when $T_{0}$ is an isosceles triangle in [21]. He claimed that the proof for the general case was rather messy, and hoped for an elegant simple proof.

(ii) Since the limiting triangles in the examples above are actually points, it might be improper to talk about the shapes of the limits of these sequences. However, we may rescale the triangles after they have been constructed. To be more specific, notice that in Example 2.1, the initial triangle $T_{0}$ is always inscribed in a circle $\Gamma$ with radius $r$. After the construction of the second triangle $T_{1}$ in terms of the "incircle" $\Gamma_{0}$ of $T_{0}$, we may rescale the circle $\Gamma_{0}$ to the same size as $\Gamma$. Consequently, the triangle $T_{1}$ will be rescaled too by a similarity transformation $S$. That is, we change the size of $T_{1}$ and preserve its shape! Continue this rescaling every time for the subsequent triangles. It is clear that we are actually dealing with a sequence of triangles that are all inscribed in the same circle (if we identify all congruent circles). See Figure 3.

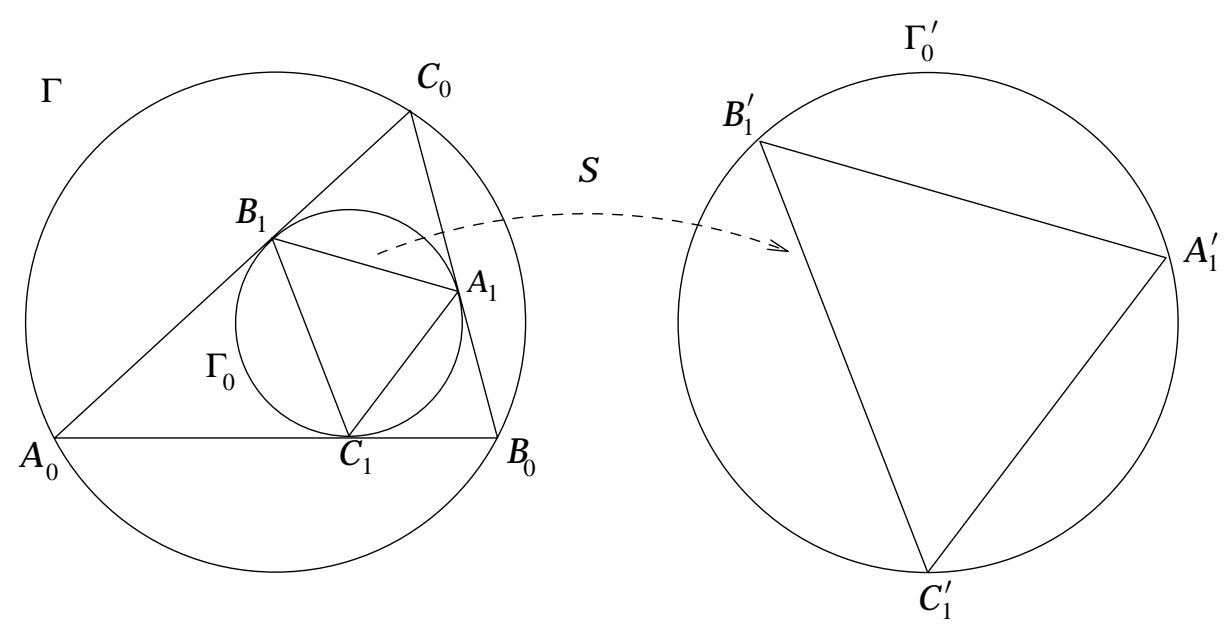

Fig. 3

(iii) From the point of view of dynamic systems, if we denote by $\mathscr{P}$ the set of all triangles that are inscribed in the circle $\Gamma$, and let $F$ be a map from $\mathscr{P}$ to itself defined by the construction and rescaling of triangles mentioned above, then the sequence of triangles can be viewed as the orbit of $T_{0}$ under the iteration of $F$. We are interested in the limiting behavior of the orbit of a given triangle. How complicated $F$ can be depends on the construction. In the next section, we will be 
concerned with constructions and rescaling of various polygons and will present the map $F$ analytically and algebraically.

\section{Sequences of Polygons}

We now turn to the general $n$-sided polygons. Let $P$ be a convex $n$-sided plane polygon and let $T P$ be the polygon whose vertices are the midpoints of the sides of $P$. We are interested in the sequence of polygons $\left\{P, T P, T^{2} P, \ldots\right\}$, and especially in the change of the shapes of these polygons. From elementary geometry, it is well-known that if $P$ is a triangle, then $T P$ is similar to $P$. Therefore, all $T^{m} P$ 's, $m=1,2, \ldots$, are similar despite their sizes. If $P$ is a quadrilateral, then $T P$ is a parallelogram, and $T^{m} P$ is similar to $T P$ if $m$ is odd, to $T^{2} P$ if $m$ is even. However, when $P$ is a convex $k$-sided polygon with $k \geq 5$, the problem is far from simple. There have been a number of articles that discussed the possible change of the shape of $T^{m} P$ with different emphases $[3,4$, $5,7,20]$. Since a sequence of polygons obtained by the midpoint-construction always converges to a point (the centroid of the initial polygon [7]) it might be awkward to talk about the "limiting figure" which is degenerate. Inspired by Remark 2.3(ii) in Section 2, we will make a slight change to the midpoint-construction. We will be concerned with only the sequences of cyclic polygons and will study the limits of these sequences.

Let $P$ be an $n$-sided polygon with vertices $z_{1}, z_{2}, \ldots, z_{n}$, inscribed in a unit circle $\Gamma$ centered at $O$. Assume that $O$ is in the interior of $P$. Join $O$ to each midpoint on the sides of $P$ and extend these line segments to meet the circle $\Gamma$ at points $v_{1}, v_{2}, \ldots, v_{n}$, respectively. These new points form a second $n$-sided polygon that is inscribed in the same circle as $P$. Denote the second polygon by $T P$ where $T$ represents a transformation on the set of all $n$-sided polygons inscribed in $\Gamma$. We are interested in the sequence of polygons $\left\{P, T P, T^{2} P, \ldots\right\}$ and the limit of $T^{m} P$ as $m \rightarrow \infty$. Since we have stretched every midpoint on the sides of $P$ to the circumference of $\Gamma$, the resulting polygons are no longer nested like those discussed in $[4,7]$. We will call the sequence of polygons $\left\{T^{m} P\right\}_{m=0}^{\infty}$ the midpoint-stretching polygons generated by $P$.

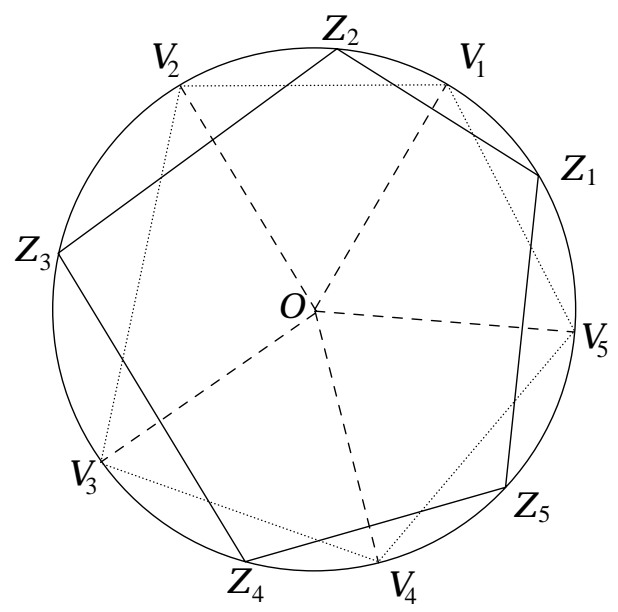

Fig. 4 
Theorem A Every sequence of midpoint-stretching polygons converges to the regular polygon.

Proof. Let $a_{i}=z_{i} z_{i+1}$ be the $i^{\text {th }}$ side of $P, i=1,2, \ldots, n$ (where $z_{n+1}=z_{1}$ ) and let $\theta_{i}$ denote the central angle of $\Gamma$ subtended by $a_{i}$ where $1 \leq i \leq n$ and $\sum_{i=1}^{n} \theta_{i}=2 \pi$. Since we are concerned only with cyclic polygons inscribed in the same circle and interested only in their shapes, we may characterize the polygon $P$ by an $n$-tuple of real numbers $\Theta=\left[\theta_{1}, \theta_{2}, \ldots, \theta_{n}\right]$ and denote by $P(\Theta)$ the corresponding congruence class of cyclic polygons. From the construction of TP whose vertices are $v_{1}, v_{2}, \ldots, v_{n}$, we see that the central angles subtended by the sides of TP are $\left(\theta_{1}+\theta_{2}\right) / 2,\left(\theta_{2}+\theta_{3}\right) / 2, \ldots,\left(\theta_{n-1}+\theta_{n}\right) / 2$. Setting

$$
T=\frac{1}{2}\left[\begin{array}{ccccc}
1 & 1 & 0 & \cdots & 0 \\
0 & 1 & 1 & \cdots & 0 \\
\vdots & \vdots & \vdots & \ddots & \\
1 & 0 & 0 & \cdots & 1
\end{array}\right]
$$

we may represent $T P$ by $P(T \Theta)$, and inductively, $T^{m} P$ by $P\left(T^{m} \Theta\right)$. Because the matrix $T$ is a doubly stochastic matrix and $\sum_{i=1}^{n} \theta_{i}=2 \pi$, it can be proved (see Section 5) that

$$
\lim _{m \rightarrow \infty}\left[T^{m} \Theta\right]=[2 \pi / n, 2 \pi / n, \ldots, 2 \pi / n] .
$$

Therefore, the sequence of midpoint-stretching polygons converges to the regular polygon.

Figure 5 shows the deformation of a cyclic 5-gon $P(\Theta)$ under the iteration of the midpoint-stretching, where $\Theta=(0.1 \pi, 0.3 \pi, 0.7 \pi, 0.55 \pi, 0.35 \pi)$

\section{Remark 3.1}

(i) Intuitively, $T$ averages every two consecutive central angles of the polygon eventually "evening up" all of them.

(ii) Doubly stochastic matrices are a very important class of matrices. We will briefly review some of their properties that are relevant to our problems in the next section.

(iii) Cyclic polygons play important roles in isoperimetric inequalities. It is well-known that among all $n$-sided plane polygons with the given set of $n$-sides, the cyclic one encloses the largest area $[13,16,23]$. Thereafter the study of the isoperimetric inequalities for plane polygons can be reduced to the study of isoperimetric inequalities for cyclic polygons. We have discussed this problem in articles [23, 24] and have introduced the "degree of irregularity" for cyclic polygons which has some probabilistic interpretations. We find the same idea can also be used in the discussion of midpoint-stretching polygons. In particular, we will prove the above limit (1) in Section 5. 

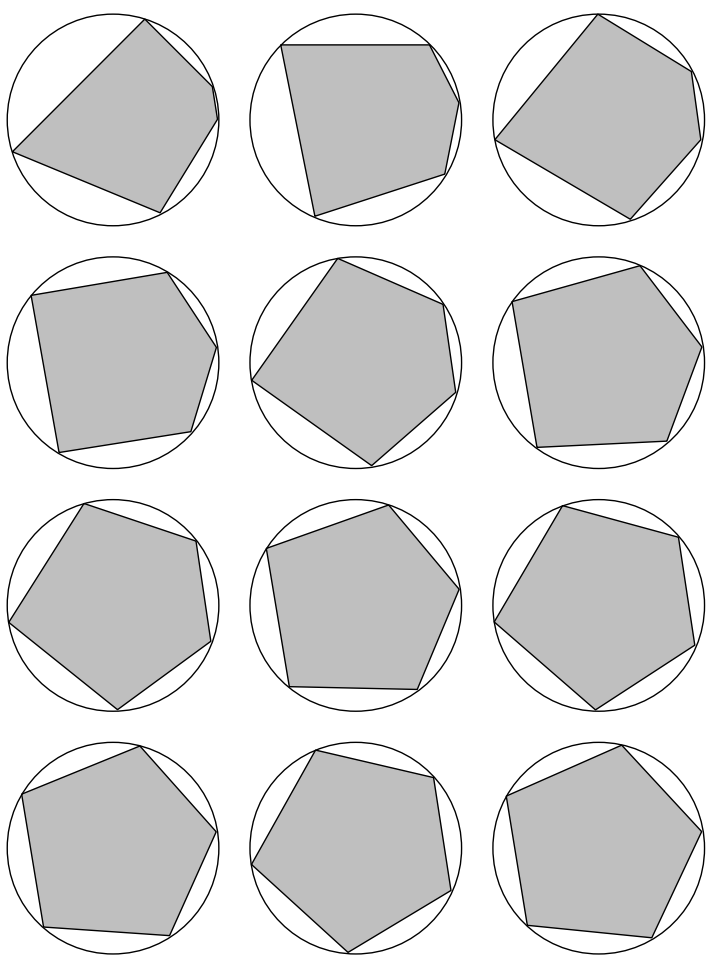

Fig. 5

In general, we choose an arbitrary point on the $i^{\text {th }}$ side of $P$ and stretch the line segment joining the center of $\Gamma$ with this point to meet the circumference of $\Gamma$ at a point $v_{i}$, $i=1,2, \ldots, n$. Denote the polygon with vertices $v_{1}, v_{2}, \ldots, v_{n}$ by $T P$, then we may characterize this polygon by the $n$-tuple of its central angles $\Phi=\left[\phi_{1}, \phi_{2}, \ldots, \phi_{n}\right]$, where

$$
\phi_{1}=\left(1-\lambda_{1}\right) \theta_{1}+\lambda_{2} \theta_{2}, \quad \phi_{2}=\left(1-\lambda_{2}\right) \theta_{2}+\lambda_{3} \theta_{3}, \ldots, \phi_{n}=\left(1-\lambda_{n}\right) \theta_{n}+\lambda_{1} \theta_{1},
$$

and each $\lambda_{i}(i=1,2, \ldots, n)$ is a real number between 0 and 1 . We may rewrite TP as $P(\Phi)=P(T \Theta)$ where the transformation $T$ can be expressed as the following matrix:

$$
T=\left[\begin{array}{ccccc}
1-\lambda_{1} & \lambda_{2} & 0 & \cdots & 0 \\
0 & 1-\lambda_{2} & \lambda_{3} & \cdots & 0 \\
\vdots & \vdots & \vdots & \ddots & \\
\lambda_{1} & 0 & 0 & \cdots & 1-\lambda_{n}
\end{array}\right]
$$

It is clear that $T$ is a doubly stochastic matrix if and only if $\lambda_{1}=\lambda_{2}=\cdots=\lambda_{n}$. Let us set $\Lambda=\left(\lambda_{1}, \lambda_{2}, \ldots, \lambda_{n}\right)$, and call the sequence of polygons constructed by the iteration of $T,\left\{T^{m} P\right\}_{m=0}^{\infty}$, the $\Lambda$-stretching polygons generated by $P$ under $T$.

Theorem B The $\Lambda$-stretching polygons converge to the regular polygon if $\lambda_{1}=\lambda_{2}=$ $\cdots=\lambda_{n}=t$ where $0<t<1$. 


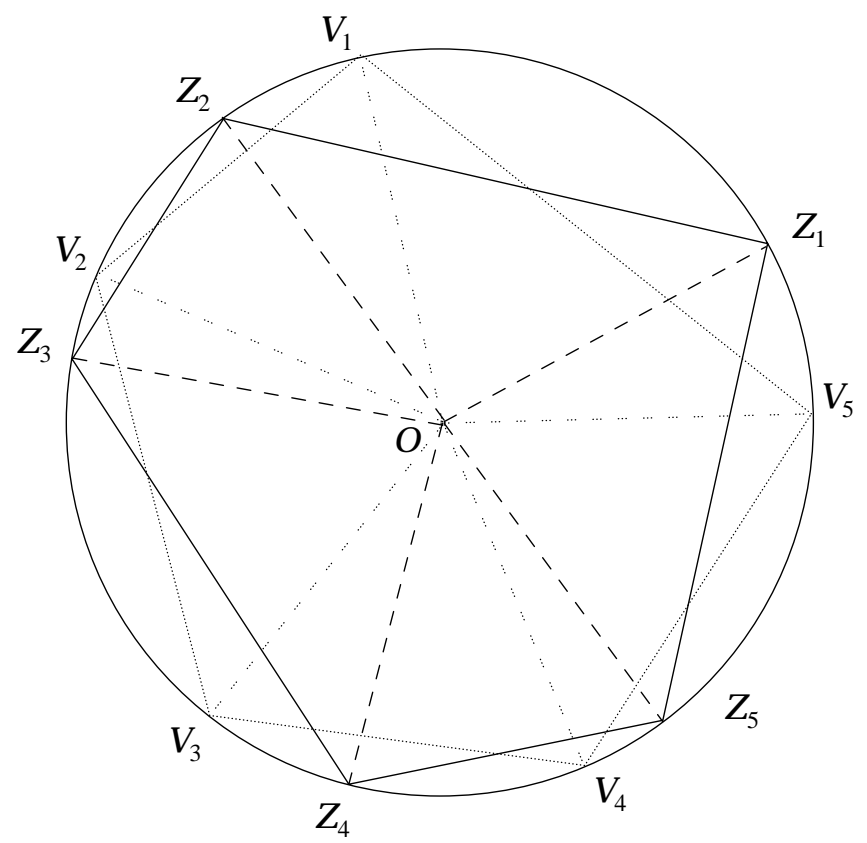

Fig. 6

Proof. See Section 5.

Note 3.2 Theorem A is simply a special case of Theorem $B$ when $t=1 / 2$. Figure 7 shows the convergence of a sequence of 6-gons $P\left(T^{m} \Theta\right)$ with

$$
\Theta=(0.25 \pi, 0.1 \pi, 0.35 \pi, 0.75 \pi, 0.45 \pi, 0.1 \pi)
$$

under the iteration of the $\Lambda$-stretching where $\Lambda=(0.27,0.27, \ldots, 0.27)$.

\section{Doubly Stochastic Matrices and Schur-Convex Functions}

In what follows, we will briefly review some basic concepts about doubly stochastic matrices and Schur-convex functions. A more detailed study of these topics and their fruitful applications can be found in $[17,19]$.

Definition 4.1 An $n \times n$ matrix $S=\left[s_{i j}\right]$ is called doubly stochastic if $s_{i j} \geq 0$ for $1 \leq i, j \leq n$, and

$$
\sum_{j=1}^{n} s_{i j}=1, \quad i=1,2, \ldots, n ; \quad \sum_{i=1}^{n} s_{i j}=1, \quad j=1,2, \ldots, n .
$$

\section{Example 4.2}

(i) A permutation matrix is a doubly stochastic matrix. (A permutation matrix is a matrix obtained by permuting the rows of the identity matrix.)

(ii) $S=\left[s_{i j}\right]$ with $s_{i j}=\frac{1}{n}, 1 \leq i, j \leq n$ is a doubly stochastic matrix. 

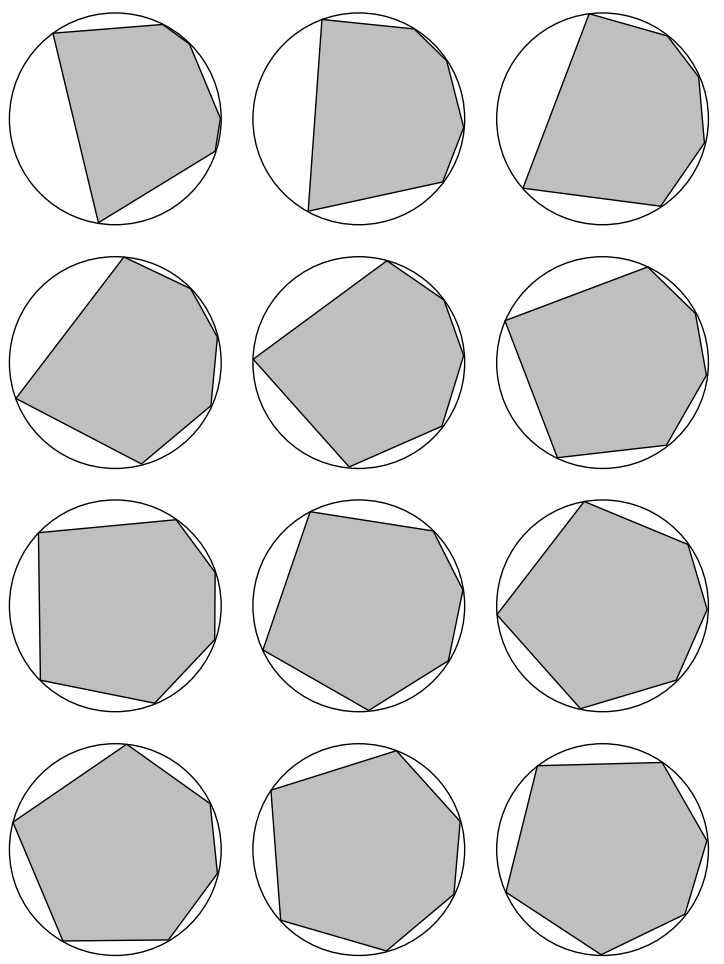

Fig. 7

Let $I^{n}=I \times I \times \cdots \times I$ ( $n$ copies), where $I$ is an interval on the real number line $\mathbb{R}$. We are interested in the following special multivariable functions.

Definition 4.3 A function $f: I^{n} \longrightarrow \mathbb{R}(n>1)$ is called Schur-convex if for every doubly stochastic matrix $S$,

$$
f(S \mathbf{x}) \leq f(\mathbf{x})
$$

for all $\mathbf{x} \in I^{n}$. It is called strictly Schur-convex if the inequality is strict and Schurconcave (respectively, strictly Schur-concave) if the inequality (2) is reversed.

Definition 4.4 A function $f: I^{n} \longrightarrow \mathbb{R}(n>1)$ is called symmetric if for every permutation matrix $P$,

$$
f(P \mathbf{x})=f(\mathbf{x})
$$

for all $\mathbf{x} \in I^{n}$.

Every Schur-convex function is a symmetric function. Because if $P$ is a permutation matrix, so is its inverse $P^{-1}$. Hence if $f$ is Schur-convex, then

$$
f(\mathbf{x})=f\left(P^{-1}(P \mathbf{x})\right) \leq f(P(\mathbf{x})) \leq f(\mathbf{x}) .
$$

This shows that $f(P \mathbf{x})=f(\mathbf{x})$ for every permutation matrix $P$, so $f$ is symmetric. On the other hand, it is not hard to see that not every symmetric function is a Schur-convex 
function (cf. [19, p. 258]). Similarly, every Schur-concave function is symmetric, but not conversely. However, we have the following so-called Schur's condition.

Lemma 4.5 ([19, p. 259]) Let $f(\mathbf{x})=f\left(x_{1}, x_{2}, \ldots, x_{n}\right)$ be symmetric and have continuous partial derivatives on $I^{n}$ where $I$ is an open interval. Then $f: I^{n} \longrightarrow \mathbb{R}$ is Schur-convex if and only if

$$
\left(x_{i}-x_{j}\right)\left(\frac{\partial f}{\partial x_{i}}-\frac{\partial f}{\partial x_{j}}\right) \geq 0
$$

on $I^{n}$. It is strictly Schur-convex if (3) is a strict inequality for $x_{i} \neq x_{j}, 1 \leq i, j \leq n$.

Since $f(\mathbf{x})$ is symmetric, Schur's condition can be reduced to ([17, p. 57])

$$
\left(x_{1}-x_{2}\right)\left(\frac{\partial f}{\partial x_{1}}-\frac{\partial f}{\partial x_{2}}\right) \geq 0,
$$

and $f$ is strictly Schur-convex if (4) is a strict inequality for $x_{1} \neq x_{2}$. The Schur's condition that guarantees a symmetric function being Schur-concave is the same as (3) or (4) except for the direction of the inequality.

Example 4.6 Let $\Theta=\left(\theta_{1}, \theta_{2}, \ldots, \theta_{n}\right)$, where $0<\theta_{i}<\pi, i=1,2, \ldots, n$ and $\sum_{i=1}^{n} \theta_{i}=$ $\pi$. Define

$$
F(\Theta)=n \sin \frac{\pi}{n}-\sum_{i=1}^{n} \sin \theta_{i},
$$

then it is easy to verify by (4) that $F$ is a strictly Schur-convex function because

$$
\left(\theta_{1}-\theta_{2}\right)\left(\frac{\partial F}{\partial \theta_{1}}-\frac{\partial F}{\partial \theta_{2}}\right)=\left(\theta_{1}-\theta_{2}\right)^{2} \sin \theta^{*}>0 \quad \text { for } \quad \theta_{1} \neq \theta_{2},
$$

where $\theta^{*}$ is between $\theta_{1}$ and $\theta_{2}$. Moreover,

$$
F(\Theta) \geq 0 \text {, with equality holding if and only if } \theta_{1}=\theta_{2}=\cdots=\theta_{n}=\pi / n .
$$

\section{Degree of Irregularity and Isoperimetric Inequalities}

Let us recall the classical isoperimetric inequality for an $n$-sided plane polygon $P_{n}$ :

$$
L_{n}^{2}-4 d_{n} A_{n} \geq 0,
$$

where $L_{n}$ is the perimeter of $P_{n}, A_{n}$ is the area of the domain enclosed by $P_{n}$, and $d_{n}=n \tan \frac{\pi}{n}$. Equality holds if and only if $P_{n}$ is regular $([13,16,18,23])$.

In geometry, we call $L_{n}^{2}-4 d_{n} A_{n}$ the isoperimetric deficit of the polygon $P_{n}$. It measures the deviation of $P_{n}$ from the "regularity". Among all $n$-sided plane polygons with given $n$ sides, the cyclic polygon encloses the largest area $([13,16,23])$. So to investigate the isoperimetric inequalities for plane polygons, we need to pay attention to cyclic polygons only. Now, for a cyclic polygon $P_{n}$ with lengths of sides $a_{1}, a_{2}, \ldots, a_{n}$, if $\theta_{1}, \theta_{2}, \ldots, \theta_{n}$ are the central angles subtended by the $n$ sides respectively, and $0<\theta_{i}<\pi$ for $i=1,2, \ldots, n$, we denote $P_{n}$ by $P_{n}(\Theta)$ where $\Theta=\left(\theta_{1}, \theta_{2}, \ldots, \theta_{n}\right), \sum_{i=1}^{n} \theta_{i}=2 \pi$, and introduce the degree of irregularity for $P_{n}(\Theta)$. 
Definition 5.1 Let $P_{n}(\Theta)$ be an $n$-sided cyclic polygon. The degree of irregularity of $P_{n}(\Theta)$ is defined as

$$
\operatorname{Deg}\left[P_{n}(\Theta)\right]=n \sin \frac{\pi}{n}-\sum_{i=1}^{n} \sin \left(\theta_{i} / 2\right)
$$

It is clear that $\operatorname{Deg}\left[P_{n}(\Theta)\right] \geq 0$ for all $P_{n}(\Theta)$, and $\operatorname{Deg}\left[P_{n}(\Theta)\right]=0$ only when $P_{n}(\Theta)$ is regular. If $P_{n}(\Theta)$ is inscribed in a circle of radius $r$, then $\operatorname{Deg}\left[P_{n}(\Theta)\right]$ times $2 r$ is simply the difference between the perimeters of $P_{n}(\Theta)$ and the regular $n$-sided polygon inscribed in the same circle. We have noticed that the equality in (6) distinguishes only the regular polygon among all isoperimetric polygons with the same number of sides. The following theorem allows us to compare any two $n$-sided cyclic polygons in terms of their degrees of irregularity.

Theorem 5.2 Let $P_{n}(\Theta)$ and $Q_{n}(\bar{\Theta})$ be two n-sided cyclic polygons. If there is a doubly stochastic matrix $S$ such that $\bar{\Theta}=S \Theta$, then

$$
\operatorname{Deg}\left[Q_{n}(\bar{\Theta})\right] \leq \operatorname{Deg}\left[P_{n}(\Theta)\right]
$$

Equality holds if and only if $\Theta$ and $\bar{\Theta}$ differ by a permutation.

Proof. Let $F(\Theta)=\operatorname{Deg}\left[P_{n}(\Theta)\right]$, then $F(\bar{\Theta})=\operatorname{Deg}\left[Q_{n}(\bar{\Theta})\right]$. From Example 4.6 we see that $F$ is Schur-convex, hence

$$
F(\bar{\Theta})=F(S \Theta) \leq F(\Theta)
$$

\section{Remark 5.3}

(i) $\operatorname{Deg}\left[P_{n}(\Theta)\right]$ is a homothetic invariant for cyclic polygons. Hence if $P_{n}(\Theta)$ and $Q_{n}(\bar{\Theta})$ are two $n$-sided homothetic cyclic polygons, that is, $\Theta=\bar{\Theta}$ (they have the same shape but may have different size), then they have the same degree of irregularity. On the other hand, $\operatorname{Deg}\left[P_{n}(\Theta)\right]=\operatorname{Deg}\left[Q_{n}(\bar{\Theta})\right]$ does not necessarily imply the homotheticity of $P_{n}(\Theta)$ and $Q_{n}(\bar{\Theta})$ because $\Theta$ and $\bar{\Theta}$ may differ by a permutation so that the two cyclic polygons may not be obtained from each other by a homothetic transformation. If we consider the class of all $n$-sided cyclic polygons $(n>3)$, the degree of irregularity can be used to classify the polygons according to their deviations from the regularity.

(ii) There are many different ways to define the degree of irregularity for a polygon. For more examples see [23, 24].

Theorem $\mathbf{C}$ Let $P(\Theta)$ be an n-sided cyclic polygon, where $\Theta=\left(\theta_{1}, \theta_{2}, \ldots, \theta_{n}\right)$ is the set of the central angles subtended by the sides of $P(\Theta)$. If $T$ is an $n$ by $n$ doubly stochastic matrix other than a permutation matrix, then $P\left(T^{m} \Theta\right)$ converges to the regular polygon as $m \rightarrow \infty$. 
Proof. Let $F(\Theta)$ denote the degree of irregularity of the initial polygon $P(\Theta)$. If the matrix $T$ is doubly stochastic other than a permutation, then

$$
F(\Theta)>F(T \Theta)>F\left(T^{2} \Theta\right)>\cdots=0 .
$$

That is, the degree of irregularity of $T^{m} P$ is monotonically decreasing and bounded from below by 0 . Therefore the limit of $\left\{F\left(T^{m} \Theta\right)\right\}_{m=0}^{\infty}$ exists, and it is not hard to see that we must have

$$
\lim _{m \rightarrow \infty} F\left(T^{m} \Theta\right)=0 .
$$

This shows that the limit of the sequence $\left\{T^{m} P\right\}$ has to be the regular polygon.

Proof of Theorem $B$. The matrix $T$ used in a $\Lambda$-stretching is a doubly stochastic matrix if all the components of $\Lambda$ are the same real number $t$, where $0<t<1$. Theorem $\mathrm{B}$ follows from Theorem $\mathrm{C}$ immediately.

\section{Remark 5.4}

(i) In fact, the degree of irregularity quantifies the shapes of cyclic polygons as the isoperimetric deficit does for general polygons. Theorems A and B provide an algorithm for changing a cyclic polygon to the regular polygon by reducing its degree of irregularity.

(ii) If we view the vertices of a cyclic polygon $P$ as a set of points $\left[z_{1}, z_{2}, \ldots, z_{n}\right]$ that are distributed on the circle at random, $\Lambda$-stretching is simply a redistribution of these points. If one continues the process of this redistribution, then these $n$ points will be evenly spread on the circle. For some probability problems that involve inscribed or circumscribed polygons, refer to [17] and references therein.

Let us recall the nesting triangles that were discussed in Section 2. Up to a rescaling of their circumscribed circles to the same size, those triangles can be viewed as special cases of sequences of cyclic $n$-sided polygons when $n=3$. Since we are concerned with only their shapes and the rescaling involves only simple similarity transformations, we may treat them uniformly by the conclusion of Theorem $\mathrm{C}$.

First of all, for Example 2.1, let $P(\Theta)$ be the initial triangle $T_{0}$ and $P(\Phi)=P(T \Theta)$ be the second triangle $T_{1}$ upon a rescaling. Then from Figure 8 it is clear that

$$
A_{0}=\theta_{1} / 2, B_{0}=\theta_{2} / 2, C_{0}=\theta_{3} / 2, \quad A_{1}=\phi_{1} / 2, B_{1}=\phi_{2} / 2, C_{1}=\phi_{3} / 2 .
$$

From the earlier observation in Section 2, we have

$$
\phi_{1}=2 A_{1}=\pi-A_{0}=\pi-\frac{\theta_{1}}{2}=\frac{\theta_{2}+\theta_{3}}{2} .
$$

Similarly, $\phi_{2}=\left(\theta_{3}+\theta_{1}\right) / 2$ and $\phi_{3}=\left(\theta_{1}+\theta_{2}\right) / 2$.

That is, the nesting triangles in Example 2.1, up to similarity transformations, are actually midpoint-stretching triangles. By Theorem A, they converge to the equilateral one.

Secondly, we give an alternate proof to Example 2.2. Although they might not be regarded as special $\Lambda$-stretching triangles, they can be viewed as a simple case of Theorem C. 


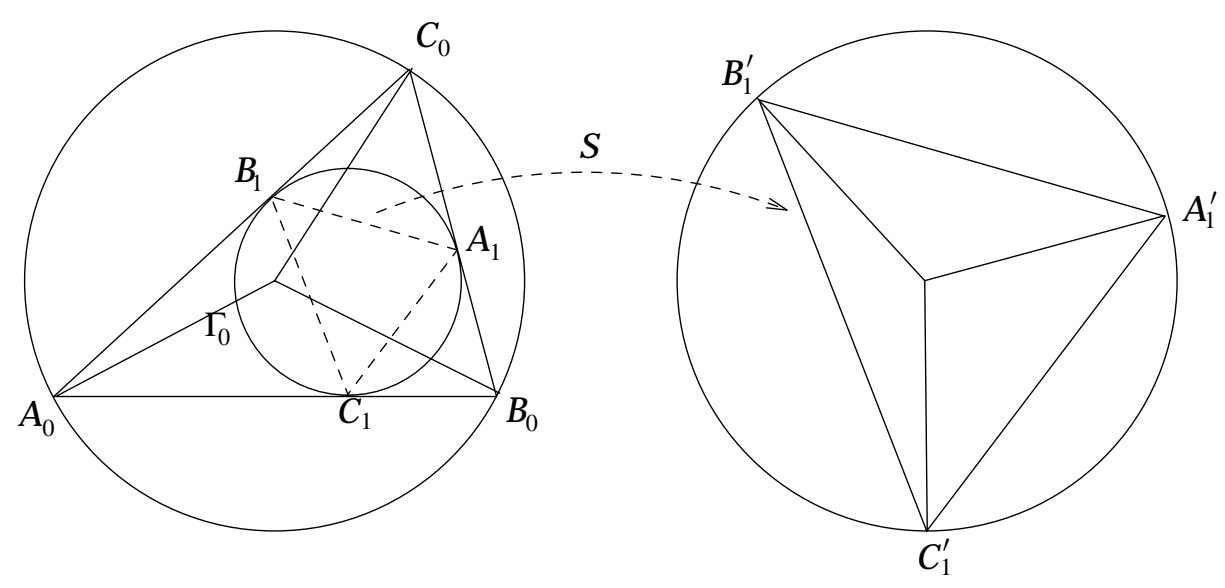

Fig. 8

Let $P(\Theta)$ be the initial triangle and $P(\Phi)=P(T \Theta)$ be the the second triangle (upon a rescaling) in Example 2.2, where $\Theta=\left(\theta_{1}, \theta_{2}, \theta_{3}\right)$ and $\Phi=\left(\phi_{1}, \phi_{2}, \phi_{3}\right)$ are the sets of central angles of the two triangles, respectively. A direct calculation shows that the matrix $T$ is the following:

$$
T=\left[\begin{array}{lll}
1 / 2 & 1 / 4 & 1 / 4 \\
1 / 4 & 1 / 2 & 1 / 4 \\
1 / 4 & 1 / 4 & 1 / 2
\end{array}\right]
$$

which is clearly a doubly stochastic matrix. Hence the limiting shape of the triangles in Example 2.2 must be an equilateral triangle by Theorem $\mathrm{C}$.

In elementary geometry, there are some equilateral triangles associated with a given triangle. Perhaps two of the most well-known examples are the so-called Morley triangle and Napoleon triangle (refer to [6, 8]). From a dynamic systems point of view, there exists a transformation $T$ acting on the set of all triangles $\{P(\Theta)\}$ such that $T P(\Theta)=P(T \Theta)$ is equilateral for any triangle $P(\Theta)$. It is clear that such a transformation $T$ is the special doubly stochastic matrix

$$
T=\left[\begin{array}{lll}
1 / 3 & 1 / 3 & 1 / 3 \\
1 / 3 & 1 / 3 & 1 / 3 \\
1 / 3 & 1 / 3 & 1 / 3
\end{array}\right]
$$

That is, we could summarize those particular transformations that change a given triangle to an equilateral one as a result of the matrix $T$ acting on the triangle.

Philip J. Davis has done an extensive study on the problems of nested polygons by using circulant matrices. Although we have approached these problems from a different perspective, all the matrices that we have used so far are circulant matrices, too. For a detailed study of circulant matrices and related geometry problems, refer to the work of Davis $[5,8,9]$. 


\section{Dynamic Properties of Cyclic Polygons}

In this section we will discuss a few interesting dynamic properties of cyclic polygons. For simplicity, let $\mathscr{P}_{n}$ be the set of all $n$-sided polygons that are inscribed in a circle $\Gamma$ of radius $r$, and let $T_{\Lambda}$ be the $\Lambda$-stretching. A polygon $P(\Theta) \in \mathscr{P}_{n}$ is called a fixed polygon of $T_{\Lambda}$ if $T_{\Lambda} P(\Theta)=P(\Theta)$ (up to a rotation about the center of $\Gamma$ ). It is called a periodic polygon with period $m$ if, up to a rotation about the center of $\Gamma, T_{\Lambda}^{m} P(\Theta)=P(\Theta)$ and $T_{\Lambda}^{k} P(\Theta) \neq P(\Theta)$ for $k=1,2, \ldots, m-1$.

Example 6.1 Let $n=3, \Theta=(3 \pi / 5,2 \pi / 3,11 \pi / 15), \Lambda=(1 / 3,1 / 2,4 / 11)$. Then the triangle $P(\Theta)$ is a fixed polygon of the transformation $T_{\Lambda}$. In fact, if we let $C$ be the matrix

$$
C=\left[\begin{array}{lll}
0 & 1 & 0 \\
0 & 0 & 1 \\
1 & 0 & 0
\end{array}\right],
$$

then $C \Theta$ represents a rotation on the components of $\Theta$. Set the transformation $T=C \circ T_{\Lambda}$. It is easy to verify that $T P(\Theta)=P(\Theta)$. In general, we have the following result.

Theorem 6.2 Every $\Lambda$-stretching transformation has at least one fixed polygon.

Proof. For a given $\Lambda=\left(\lambda_{1}, \lambda_{2}, \ldots, \lambda_{n}\right)$, if all $\lambda_{i}$ 's are equal, where $0<\lambda_{i}<1$ and $1 \leq i \leq n$, then the regular polygon is its fixed polygon and any other polygons will converge to the regular one by Theorem B. Suppose these $\lambda_{i}$ 's are different and consider the following $(n+1) \times(n+1)$ system of linear equations

$$
\begin{aligned}
\theta_{1}+\cdots+\theta_{n} & =2 \pi \\
\left(1-\lambda_{1}\right) \theta_{1}+\lambda_{2} \theta_{2}-\theta_{3} & =0 \\
\left(1-\lambda_{2}\right) \theta_{2}+\lambda_{3} \theta_{3}-\theta_{4} & =0 \\
\cdots & =0 \\
-\theta_{1}+\left(1-\lambda_{n-1}\right) \theta_{n-1}+\lambda_{n} \theta_{n} & =0 \\
\lambda_{1} \theta_{1}-\theta_{2}+\left(1-\lambda_{n}\right) \theta_{n} & =0 .
\end{aligned}
$$

From the coefficient matrix $A$ of the last $n$ equations where

$$
A=\left[\begin{array}{ccccccc}
1-\lambda_{1} & \lambda_{2} & -1 & 0 & \cdots & 0 & 0 \\
0 & 1-\lambda_{2} & \lambda_{3} & -1 & \cdots & 0 & 0 \\
\vdots & \vdots & \vdots & \vdots & \vdots & \vdots & \vdots \\
-1 & 0 & 0 & 0 & \cdots & 1-\lambda_{n-1} & \lambda_{n} \\
\lambda_{1} & -1 & 0 & 0 & \cdots & 0 & 1-\lambda_{n}
\end{array}\right]
$$

we see that $\operatorname{rank}(A)<n$ since the sum of its $n$ rows is a 0 row. Therefore, the last $n$ homogeneous linear equations have non-trivial solutions with at least one free variable. Substituting them into the first equation we get a solution for that system. Geometrically, the system represents a $\Lambda$-stretching transformation $T$ such that $\Phi=\left(\phi_{1}, \phi_{2}, \ldots, \phi_{n}\right)=$ $T \Theta$ is obtained by rotating the components of $\Theta$. 
Remark 6.3 Unlike the study of ordinary matrix iterations in which the eigenvalues and eigenvectors play the essential roles when one tries to compute $T^{m}$, our transformation $T$ is supposed to act on the $n$-tuple $\Theta=\left(\theta_{1}, \ldots, \theta_{n}\right)$ whose components are constrained by the condition $\sum_{i=1}^{n} \theta_{i}=2 \pi$. Thus $T \Theta=\mu \Theta$ yields $\mu=1$ since we must have $\sum_{i=1}^{n} \mu \theta_{i}=2 \pi$.

In general, let $P(\Theta)$ be an $n$-sided cyclic polygon with the set of central angles $\Theta=$ $\left(\theta_{1}, \theta_{2}, \ldots, \theta_{n}\right)$, and let $T=\left[t_{i j}\right]$ be an $n \times n$ matrix acting on $\Theta$. Since

$$
\sum_{i=1}^{n} \theta_{i}=2 \pi \quad \text { and } \quad \sum_{i=1}^{n}\left(\sum_{j=1}^{n} t_{i j} \theta_{j}\right)=2 \pi
$$

a necessary condition for $T P(\Theta)=P(T \Theta)$ to be well-defined is

$$
\sum_{i=1}^{n} t_{i j}=1, \quad j=1,2, \ldots, n .
$$

That is, the sum of the entries in each column of $T$ must be 1 . When $T$ is a doubly stochastic matrix, we have seen that $T^{m} P(\Theta)=P\left(T^{m} \Theta\right)$ converges to the regular polygon for any given cyclic polygon $P(\Theta)$, and the only fixed polygon of $T$ is the regular polygon. When $T$ is a $\Lambda$-stretching transformation, then the proof of Theorem 6.2 shows that $T$ could have many different fixed polygons. However, if we only assume that $T$ satisfies equation (8), then the dynamic behavior of $\left\{T^{m} P(\Theta)\right\}_{m=0}^{\infty}$ could be very complex. To illustrate this and to conclude this paper, we will briefly mention the well-known sequence of pedal triangles.

Let $P_{0}$ be a triangle with inner angles $A_{0}, B_{0}$, and $C_{0}$. Construct a second triangle $P_{1}$ whose vertices are the feet of the altitudes of $P_{0}$ and with inner angles $A_{1}, B_{1}$, and $C_{1}$. Construct a third triangle $P_{2}$ in the same way, and so on. One obtains a sequence of triangles $\left\{P_{m}\right\}_{m=0}^{\infty}$ called pedal triangles that were studied more than a century ago [11]. In late 1980's, Kingston and Synge revisited this problem [14]. They discovered many surprising properties of such sequences and also fixed some errors that occurred in the earlier literature. The limiting shape of the sequence $\left\{P_{m}\right\}_{m=0}^{\infty}$ appeared to be almost any shape of triangle if one chooses an appropriate initial triangle $P_{0}$. Soon after their work, a number of articles made nice connections between the sequence of pedal triangles and symbolic dynamic systems and ergodic theory [2, 15, 22]. Since triangles are always cyclic, if we are concerned only with the shape of its limit, we may treat the sequence of pedal triangles as a particular case of sequences of cyclic polygons with necessary rescaling. From [14], it is known that if $P_{0}$ is an acute triangle, then the inner angles of $P_{1}$ are given by

$$
A_{1}=\pi-2 A_{0}, \quad B_{1}=\pi-2 B_{0}, \quad C_{1}=\pi-2 C_{0} .
$$

If $P_{0}$ is an obtuse triangle, say, $\pi>A_{0}>\pi / 2$, then

$$
A_{1}=2 A_{0}-\pi, \quad B_{1}=2 B_{0}, \quad C_{1}=2 C_{0} .
$$


Therefore, if $\Theta=\left(\theta_{1}, \theta_{2}, \theta_{3}\right)$ and $\Phi=\left(\phi_{1}, \phi_{2}, \phi_{3}\right)$ are the sets of central angles of $P_{0}$ and $P_{1}$ respectively, then we have:

(i) if the center of the circumscribed circle is inside $P_{0}$ (it is an acute triangle), then

$$
\phi_{1}=-\theta_{1}+\theta_{2}+\theta_{3}, \quad \phi_{2}=\theta_{1}-\theta_{2}+\theta_{3}, \quad \phi_{3}=\theta_{1}+\theta_{2}-\theta_{3} ;
$$

that is, $P_{1}=T P_{0}=P(T \Theta)$ where

$$
T=\left[\begin{array}{ccc}
-1 & 1 & 1 \\
1 & -1 & 1 \\
1 & 1 & -1
\end{array}\right]
$$

(ii) if the center of the circumscribed circle is outside of $P_{0}$ (it is an obtuse triangle), then

$$
\phi_{1}=\theta_{1}-\theta_{2}-\theta_{3}, \quad \phi_{2}=2 \theta_{2}, \quad \phi_{3}=2 \theta_{3} ;
$$

that is, $P_{1}=S P_{0}=P(S \Theta)$ where

$$
S=\left[\begin{array}{ccc}
1 & -1 & -1 \\
0 & 2 & 0 \\
0 & 0 & 2
\end{array}\right]
$$

In general, we cannot represent the pedal triangles as iterations of a single transformation matrix. For instance, if $A_{0}=36^{\circ}, B_{0}=72^{\circ}$, and $C_{0}=72^{\circ}\left(\theta_{1}=72^{\circ}, \theta_{2}=144^{\circ}\right.$, $\theta_{3}=144^{\circ}$ ), we use the matrix $T$ to obtain the second triangle $P_{1}=P(T \Theta)$. But notice that $A_{1}=108^{\circ}, B_{1}=36^{\circ}$, and $C_{1}=36^{\circ}$, and we have to use the matrix $S$ to get the third triangle $P_{2}$ because $P_{1}$ is an obtuse triangle! Using two different matrices to describe the limiting shape of a sequence of pedal triangles causes a more chaotic situation. See [14] for more details. That is why we have limited our previous discussion about sequences of cyclic polygons to some rather special cases involving only doubly stochastic matrices and $\Lambda$-stretching. However, inspired by the abstract treatment of the sequence of pedal triangles in terms of symbolic dynamic systems in [2], it seems very likely that one could approach the problem of sequences of cyclic polygons from a more abstract point of view and generalize our discussion here further to include more complicated situations. Even within the scope of the $\Lambda$-stretching transformation, there are still some questions to be answered, such as what conditions on $T$ or $\Theta$ would ensure a convergent sequence $\left\{T^{m} P(\Theta)\right\}_{m=0}^{\infty}$ (not necessarily having a regular polygon as the limit)? When does it have chaotic behavior? How can the appropriate definition of entropy be introduced? These questions seem to be interesting in geometry, algebra, and dynamic systems. On the other hand, by virtue of computer graphics, it should not be too difficult to simulate a $\Lambda$-stretching construction on a computer and to visualize the convergent or chaotic transformations generated by iterations of $T_{\Lambda}$. We will leave these questions to the interested reader and hope that the ideas and examples illustrated in this paper will bring a deeper and broader discussion on the dynamic geometry of polygons on the surfaces of constant curvature. 


\section{References}

[1] St. Abbot, Average sequences and triangles, Math. Gaz. 80 (1996), 222-224.

[2] J.C. Alexander, The symbolic dynamics of the sequence of pedal triangles, Math. Mag. 66 (1993), no. 3, $147-158$.

[3] F. Bachmann and E. Schmidt, n-gons, University of Toronto Press, Toronto, Ont., 1975, Translated from the German by Cyril W.L. Garner, Mathematical Expositions, No. 18.

[4] E.R. Berlekamp, E.N. Gilbert, and F.W. Sinden, A polygon problem, Amer. Math. Monthly 72 (1965), 233-241.

[5] Geng Zhe Chang and P.J. Davis, Iterative processes in elementary geometry, Amer. Math. Monthly 90 (1983), no. 7, 421-431.

[6] Geng Zhe Chang and T.W. Sederberg, Over and over again, New Mathematical Library, no. 39, Mathematical Association of America, Washington, DC, 1997.

[7] R.J. Clarke, Sequences of polygons, Math. Mag. 52 (1979), no. 2, 102-105.

[8] P.J. Davis, Cyclic transformations of polygons and the generalized inverse, Canad. J. Math. 29 (1977), no. 4, 756-770.

[9] P.J. Davis, Circulant matrices, John Wiley \& Sons, New York-Chichester-Brisbane, 1979, A WileyInterscience Publication, Pure and Applied Mathematics.

[10] J. Garfunkel, Problem e2906, Amer. Math. Monthly 88 (1981), no. 8, 620.

[11] E.W. Hobson, A treatise on plane geometry, Cambridge University Press, 1897.

[12] St. Jones, Two iteration examples, Math. Gaz. 74 (1990), 58-62.

[13] N.D. Kazarinoff, Geometric inequalities, New Mathematical Library, no. 4, Mathematical Association of America, Washington, DC, 1961.

[14] J.G. Kingston and J.L. Synge, The sequence of pedal triangles, Amer. Math. Monthly 95 (1988), no. 7 , 609-620.

[15] P.D. Lax, The ergodic character of sequence of pedal triangles, Amer. Math. Monthly 97 (1990), no. 5, 377-381.

[16] D.S. Macnab, Cyclic polygons and related questions, Math. Gaz. 65 (1981), no. 431, 22-28.

[17] A.W. Marshall and I.Olkin, Inequalities: theory of majorization and its applications, Academic Press Inc. [Harcourt Brace Jovanovich Publishers], New York, 1979.

[18] R. Osserman, The isoperimetric inequality, Bull. Amer. Math. Soc. 84 (1978), no. 6, 1182-1283.

[19] A.W. Roberts and D.E. Varberg, Convex functions, Academic Press [A subsidiary of Harcourt Brace Jovanovich, Publishers], New York-London, 1973, Pure and Applied Mathematics, Vol. 57.

[20] I.J. Schoenberg, Mathematical time exposures, Mathematical Association of America, Washington, D.C., 1982.

[21] S.Y. Trimble, The limiting case of triangles formed by angle bisectors, Math. Gaz. 80 (1996), no. 489, 554-556.

[22] P. Ungar, Mixing property of the pedal mapping, Amer. Math. Monthly 97 (1990), no. 10, 898-900.

[23] Xin-Min Zhang, Bonnesen-style inequalities and pseudo-perimeters for polygons, J. Geom. 60 (1997), no. 1-2, 188-201.

[24] Xin-Min Zhang, Schur-convex functions and isoperimetric inequalities, Proc. Amer. Math. Soc. 126 (1998), no. 2, 461-470.

L. Richard Hitt

Department of Mathematics and Statistics University of South Alabama

Mobile, AL 36688

USA

e-mail: hitt@mathstat.usouthal .edu
Xin-Min Zhang

Department of Mathematics and Statistics University of South Alabama

Mobile, AL 36688

USA

e-mail: zhang@mathstat.usouthal.edu 